$1-3-2022$

\title{
A New Goodness of Fit Measure Based on Income Inequality Curves
}

Shahryar Mirzaei

Payam Noor University, Tehran, Iran, shahriar_mirzaee54@yahoo.com

S. M. A. Jahanshahi

University of Sistan and Baluchestan, Zahedan, Iran, mehdijahanshahi@yahoo.com

Follow this and additional works at: https://digitalcommons.wayne.edu/jmasm

Part of the Applied Statistics Commons, Social and Behavioral Sciences Commons, and the Statistical Theory Commons

\section{Recommended Citation}

Mirzaei, Shahryar and Jahanshahi, S. M. A. (2022) "A New Goodness of Fit Measure Based on Income Inequality Curves," Journal of Modern Applied Statistical Methods: Vol. 19 : Iss. 1 , Article 24.

DOI: $10.22237 / j m a s m / 1619482080$

Available at: https://digitalcommons.wayne.edu/jmasm/vol19/iss1/24

This Regular Article is brought to you for free and open access by the Open Access Journals at DigitalCommons@WayneState. It has been accepted for inclusion in Journal of Modern Applied Statistical Methods by an authorized editor of DigitalCommons@WayneState. 


\section{A New Goodness of Fit Measure Based on Income Inequality Curves}

\author{
Shahryar Mirzaei \\ Payam Noor University \\ Tehran, Iran
}

\author{
S. M. A. Jahanshahi \\ University of Sistan and Baluchestan \\ Zahedan, Iran
}

This paper uses inequality-measurement techniques to assess goodness of fit in income distribution models. It exposes the shortcomings of the use of conventional goodness of fit criteria in face of the big income data and proposes a new set of metrics, based on income inequality curves. In this note, we mentioned that the distance between theoretical and empirical inequality curves can be considered as a goodness of fit criterion. We demonstrate certain advantages of this measure over the other general goodness of fit criteria. Unlike other goodness of fit measures, this criterion is bounded. It is 0 in minimum difference and 1 in maximum distance. Furthermore, there is a consistency between this new goodness of fit measure and the other conventional criteria. A simulation study based on fitted distribution to real income data is performed in order to investigate some statistical properties of the new goodness of fit measure. An empirical study and comparisons are also provided.

Keywords: Goodness of fit measure, income distribution, Lorenz curve, Zenga index

\section{Introduction}

Most statistical methods suppose an underlying distribution in the calculation of their results and inferences. Therefore, methods for checking that the underlying distribution have a special form, i.e., goodness of fit (GOF) tests, has been largely studied, becoming a milestone of statistics. During the last few decades, this subject has played an important role in many branches of sciences, mainly in engineering, management, and economics sciences studies. Indeed, the goal of the GOF tests is to check whether the underlying probability distribution, from which a sample is drawn, differs from a hypothesized distribution. Therefore, GOF tests determine if the empirical distribution of the data satisfies the assumptions of theoretical models.

doi: 10.22237/jmasm/1619482080 | Accepted: December 14, 2018; Published: January 3, 2022.

Correspondence: S. M. A. Jahanshahi, mjahan@math.usb.ac.ir 


\section{MIRZAEI \& JAHANSHAHI}

As is well known, in mathematics, the notion of distance is one of the most fundamental concepts. From the scientific and mathematical point of view, distances defined as a quantitative degree of how far apart two objects are. Nowadays, this concept has also an eminent role in almost all branches of science and technology including probability theory and applied statistics. Distance measures are essential to solve many pattern recognition problems such as GOF problems. GOF measures naturally arises to account for the distances between the empirical and assumed theoretical distributions.

Various distance measures are applicable to compare empirical and theoretical distribution functions. The Kolmogorov-Smirnov, Cramer-von Mises, Kuiper, and Anderson-Darling test statistics are examples of such measures (see Cramér, 1928; Watson, 1961; Marsaglia et al., 2003 for further illustrative about these criteria).

In the study of income data especially in large sample, it should be noted that all models must be rejected at conventional levels of significance. This result, not uncommon in applications involving large sample sizes, raises questions about the power of these tests (McDonald \& Xu, 1995). In such cases, the common GOF test statistic is either very large or very small (see Akaike, 1974; Cordeiro et al., 2015). Reporting such values is not interesting in scientific reports.

The use of income inequality indices in the context of GOF test has been less considered. Ascher (1990) used a wide selection of tests for exponentially based on income inequality indices and compared. Jammalamadaka and Goria (2004) introduced a test of goodness-of-fit based on Gini's index of spacings (Gini, 1921). Noughabi et al. (2014) introduced a general goodness-of-fit test based on the estimated Gini inequality index.

In this paper, we propose a set of GOF measures using income inequality curves which take into account the particular nature of income distributions models. The use of these curves in econometrics has been new and can be increased in its applications widely. Although these distance measures have not been introduced for exactly the same purpose, they have the common property of increasing as the two distributions involved move apart. Unlike other GOF measures, these criteria are bounded. They are 0 in minimum difference and 1 in maximum distance. The results show that the consistency between these new GOF measures and the other popular GOF criteria.

The paper is structured as follows. In the following section, we review the concepts of income inequality indices. We also present the sampling estimator of the traditional Lorenz curve and the new Zenga inequality curve. We then perform

GOF using the new proposed measure and compares it with standard measures in 


\section{A NEW GOODNESS OF FIT MEASURE}

the literature. A simulation study to compare the performance of the new measures is then constructed. The last section is devoted to a brief conclusion.

\section{Income Inequality Curves}

The principal curves proposed for inequality measurement are the well-known Lorenz (1905) curve, the Bonferroni (1930), and the Zenga (1984, 2007) curves. Here, we concentrate on traditional Lorenz curve and the new inequality curve proposed by Zenga (2007).

Let $x_{1}, \ldots, x_{n}$ be an ordered vector of non-negative values representing the $x_{1} \leq x_{2} \leq \ldots \leq x_{n}$ distribution of income. Lorenz (1905) introduced an inequality curve $\mathrm{L}(p)$ based on the ratio between the partial sum and the overall sum of nonnegative income by

$$
\mathrm{L}(p)=\frac{\sum_{i=1}^{[n p]} x_{i}}{\sum_{i=1}^{n} x_{i}}, \quad p \in[0,1]
$$

The Lorenz function indicates the cumulative percentage of total income held by an $\mathrm{L}(p)$ cumulative proportion $p$ of the population.

Zenga (2007) introduced a new inequality curve $Z(p)$ based on the ratio between the lower mean $\mathrm{M}^{-}(p)$ and the upper mean $\mathrm{M}^{+}(p)$ of non-negative income by

$$
\mathrm{Z}(p)=1-\frac{\mathrm{M}^{-}(p)}{\mathrm{M}^{+}(p)}, \quad p \in(0,1),
$$

where

$$
\mathbf{M}^{-}(p)=\frac{\sum_{i=1}^{[n p]} x_{i}}{[n p]} \text { and } \mathbf{M}^{+}(p)=\frac{\sum_{i=[n p]+1}^{n} x_{i}}{n-[n p]}
$$

It should be noted that by averaging the inequality curves the inequality measures are obtained. For example, the new inequality Zenga index is given by 


$$
\hat{Z}=1-\frac{1}{n-1} \sum_{i=1}^{n-1} \frac{\frac{1}{i} \sum_{j=1}^{i} X_{j: n}}{\frac{1}{n-1} \sum_{j=i+1}^{n} X_{j: n}}
$$

Moreover, these curves and synthetic measures have been extended to the continuous variables.

Definition. Let $X$ be a non-negative continuous random variable, with positive and finite expected value. The Lorenz curve of $X$ is defined as

$$
\mathrm{L}(p)=\frac{1}{\mathrm{E}(X)} \int_{0}^{p} \mathrm{Q}(u) d u, \quad p \in(0,1)
$$

where $Q($.$) is the quantile function of distribution function F$.

From such $Z(p)$ curve, the related inequality index $Z$ is defined as

$$
Z=\int_{0}^{1} \mathrm{Z}(p) d p .
$$

Zenga (2007) also showed the link between the $\mathrm{Z}(p)$ curve and the $\mathrm{L}(p)$ curve:

$$
\mathrm{Z}(p)=\frac{p-\mathrm{L}(p)}{p[1-\mathrm{L}(p)]}, \quad p \in(0,1) .
$$

The Zenga and Lorenz curves display interesting graphical representation to show inequality in income models (see Arcagni \& Porro, 2014). The Zenga curve is a flexible curve. The shape of this curve neither has forced values at the endpoints of its domain of definition nor is constrained to being non-decreasing and concave on the interval [0, 1], as is the case for the Lorenz curve (see Zenga, 2007; Polisicchio \& Porro, 2009 for further illustrative examples).

\section{The New GOF Measure}

In this section, we perform a GOF tool for some well-known income distributions by using the inequality curves. Then, we shall report the results which compares 


\section{A NEW GOODNESS OF FIT MEASURE}

the proposed measure with the common GOF measures. For more illustration, we refer to the ungrouped real income data set with 14,827 observations which is generated from real Austrian European Union Statistics on Income and Living Conditions (EU-SILC) data from 2006.

For comparison the good behavior of income distributions, we also fit the following income models to total money income of the EU-SILC data.

- The Weibull (W) distribution with density function

$$
\mathrm{f}(x)=\frac{\alpha}{\beta}\left(\frac{x}{\beta}\right)^{\alpha-1} e^{-\left(\frac{x}{\beta}\right)^{\alpha}}, \quad \beta>0, \quad \alpha>0, \quad x>0
$$

- The log-normal (LN) distribution with density function

$$
f(x)=\frac{1}{x \sqrt{2 \pi \sigma^{2}}} \exp \left\{\frac{-1}{2 \sigma^{2}}(\log (x)-\mu)^{2}\right\}, \quad-\infty<\mu<\infty, \quad \sigma^{2}>0, \quad x>0 .
$$

- The log-logistic (LL) distribution with density function

$$
f(x)=\frac{\frac{a}{b}\left(\frac{x}{b}\right)^{a-1}}{\left[1+\left(\frac{x}{b}\right)^{a}\right]^{2}}, \quad a>0, \quad b>0, \quad x>0
$$

The aforementioned distributions have been chosen because they have the same number of parameters and the same support. Also, they are the most used models for representing the income in the literature.

Here, the performance of these distributions for modelling EU-SILC income data are compared. In order to compare the distributions, we consider the following information criteria (which are considered by many researchers including Akaike, 1974; Cordeiro et al., 2015): Akaike Information Criterion (AIC), Bayesian Information Criterion (BIC), Hannan-Quinn Information Criterion (HQIC), and Consistent Akaike Information Criterion (CAIC). These statistics are given by 


\section{MIRZAEI \& JAHANSHAHI}

$$
\begin{aligned}
A I C & =-2 \hat{\ell}+2 k, \quad B I C=-2 \hat{\ell}+k \log (n), \\
H Q I C & =-2 \hat{\ell}+2 k \log [\log (n)], \quad C A I C=-2 \hat{\ell}+\frac{2 k n}{(n-k-1)}
\end{aligned}
$$

where $\hat{\ell}$ denotes the log-likelihood function evaluated at the MLEs, $k$ is the number of model parameters, and $n$ is the sample size. The model with the lowest values for these statistics could be chosen as the best model to fit the data.

Table 1 provides the maximum-likelihood estimates on real data for the parameters of the selected models. The values of Gini and Zenga indices also are reported. The last four columns contain the information criteria as GOF measures. In general, the smaller the values of these criteria, the better fit the data. The lowest value of GOF indices are reported in bold type.

The results of Table 1 show that since the values of GOF measures are smaller for LL model compared to the two other distributions, then the LL model performs better fit than LN and W models in terms of information criterion GOF measures. The LN model is the second best of three models. The $\mathrm{W}$ is, with more information criterion values, worse than any of other models. Note that the actual Gini and Zenga inequality indices for considered EU-SILC data are 0.2627 and 0.5872 , respectively. All of the theoretical models considered, have larger estimated Gini and Zenga indices, and suggest greater dispersion than empirical real income data. The Gini and Zenga indices of the fitted models are higher than the actual ones. Note that the LL model also makes the theoretical values closer to the actual ones rather than the $\mathrm{W}$ and $\mathrm{LN}$ models. The inequality indices of the estimated model are obtained through numerical procedures.

Table 1. Parameter estimates, income measures, GOF statistics for fitted models to EU-

\begin{tabular}{|c|c|c|c|c|c|c|c|c|}
\hline \multirow[b]{2}{*}{ Model } & & & \multicolumn{2}{|c|}{$\begin{array}{c}\text { Income } \\
\text { measures }\end{array}$} & \multicolumn{4}{|c|}{ GOF criteria } \\
\hline & Paramete & estimates & Gini & Zenga & AIC & BIC & HQIC & CAIC \\
\hline W & $\hat{\alpha}=2.012$ & $\hat{\beta}=22472$ & 0.3026 & 0.6318 & 314018 & 314018 & 314016 & 314018 \\
\hline LN & $\hat{\mu}=9.771$ & $\hat{\sigma}=0.549$ & 0.2914 & 0.6293 & 313483 & 313498 & 313488 & 313484 \\
\hline $\begin{array}{r}\mathrm{LL} \\
\text { Empirical }\end{array}$ & $\hat{a}=3.570$ & $\hat{b}=17950$ & $\begin{array}{l}0.2791 \\
0.2627\end{array}$ & $\begin{array}{l}0.6006 \\
0.5872\end{array}$ & 311657 & 311672 & 311655 & 311657 \\
\hline
\end{tabular}
SILC data 


\section{A NEW GOODNESS OF FIT MEASURE}
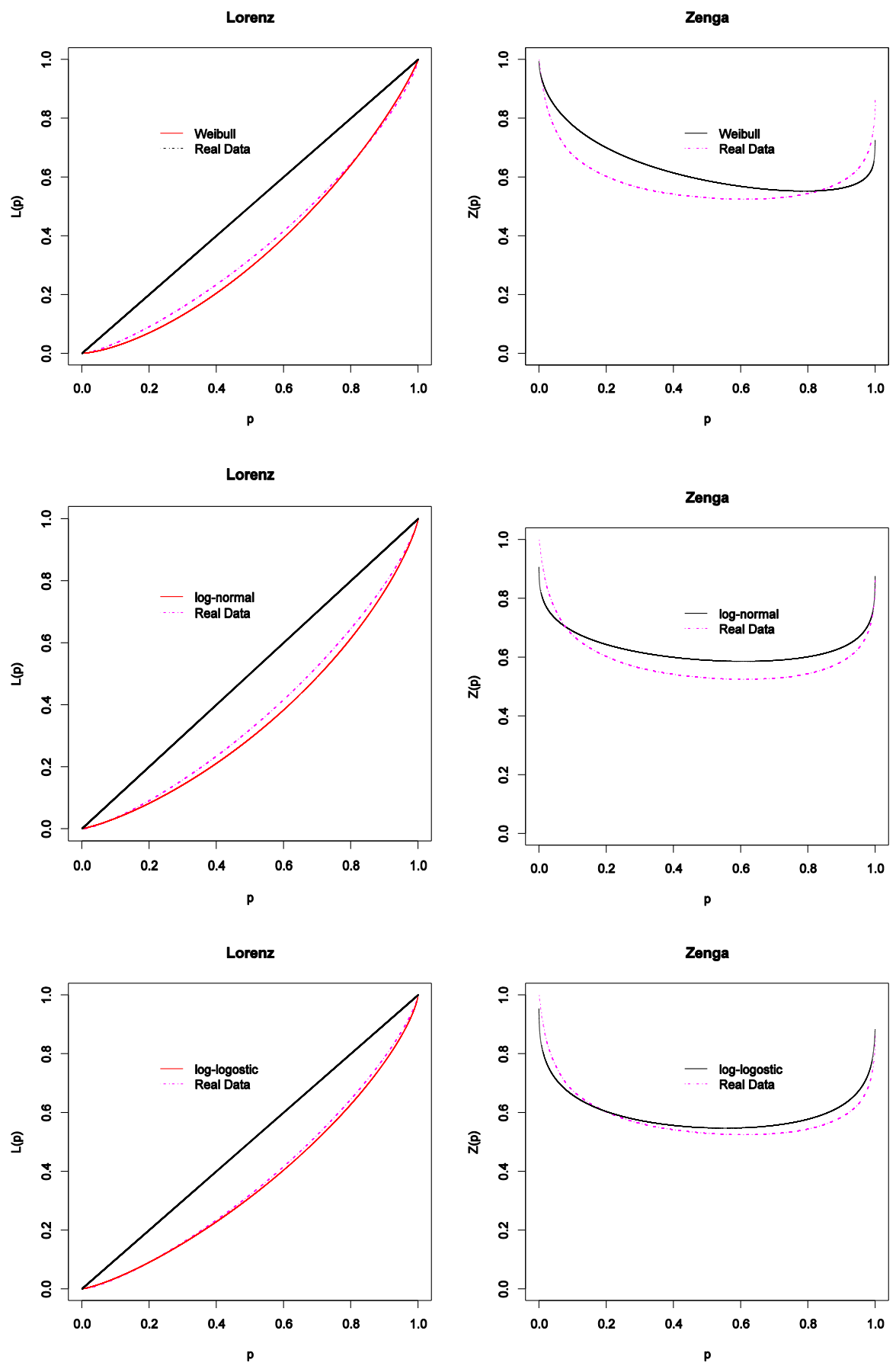

Figure 1. Lorenz and Zenga curves of Weibull, log-normal, and log-logistic models for EU-SILC data 


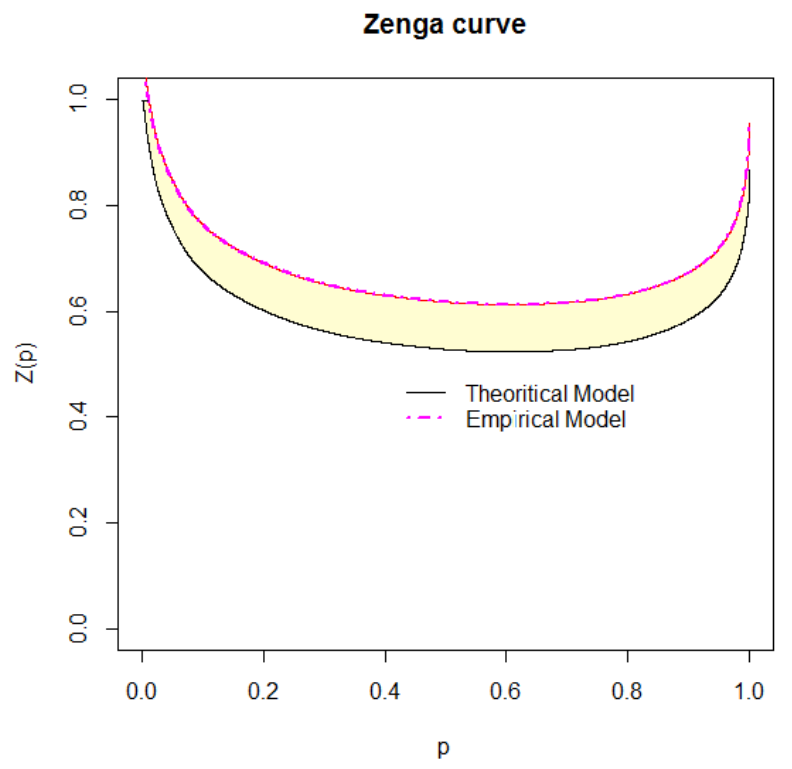

Figure 2. Distance between Zenga curves as a GOF measure

The behavior of the Zenga curves show that the theoretical models overestimate the inequality for low incomes and underestimate the inequality for high incomes for all of considered models. Remarks of such kind are difficult to be observed in the Lorenz curve. In other words, differences between the inequality curves of the theoretical and empirical models are not significant and observable in Lorenz curve. For this reason, the Zenga curve seems to be a valid alternative to the traditional Lorenz curve.

Here, the basic question is to find a way to measure the distance between theoretical and empirical Zenga curves as a GOF measure. In the right panel of Figure 2, from a geometric point of view, the new measure can be considered as the area between the empirical and the theoretical Zenga curves. This index is 0 in minimum difference and 1 in maximum distance.

A natural distance measure between empirical and theoretical Zenga curves (as shown in Figure 2) can be defined as

$$
B=\int_{0}^{1}|\hat{\mathrm{Z}}(p)-\mathrm{Z}(p)| d p,
$$

where $Z(p)$ is the empirical Zenga curve and $\hat{Z}(p)$ is the theoretical Zenga curve. 


\section{A NEW GOODNESS OF FIT MEASURE}

Table 2. The new GOF criteria for fitted models to EU-SILC income data

\begin{tabular}{rrrrr} 
Model & $\boldsymbol{B}_{\mathbf{1}}$ & $\boldsymbol{B}_{\mathbf{2}}$ & $\boldsymbol{B}_{\mathbf{3}}$ & $\boldsymbol{B}_{\mathbf{4}}$ \\
\hline W & 0.098 & 0.089 & 0.110 & 0.099 \\
LN & 0.088 & 0.081 & 0.094 & 0.086 \\
LL & $\mathbf{0 . 0 4 1}$ & $\mathbf{0 . 0 4 0}$ & $\mathbf{0 . 0 4 7}$ & $\mathbf{0 . 0 4 6}$ \\
\hline
\end{tabular}

Since this measure cannot be used to make comparisons across several populations that have different units of measurement, we can define other relative standardized measures to evaluate the GOF as

$$
\begin{aligned}
& B_{1}=\int_{0}^{1} \frac{|\hat{\mathrm{Z}}(p)-\mathrm{Z}(p)|}{\mathrm{Z}(p)} d p, \quad B_{2}=\int_{0}^{1} \frac{|\hat{\mathrm{Z}}(p)-\mathrm{Z}(p)|}{\hat{\mathrm{Z}}(p)} d p, \\
& B_{3}=\left[\int_{0}^{1}\left[\frac{\hat{\mathrm{Z}}(p)-\mathrm{Z}(p)}{\mathrm{Z}(p)}\right]^{2} d p\right]^{\frac{1}{2}}, \quad B_{4}=\left[\int_{0}^{1}\left[\frac{\hat{\mathrm{Z}}(p)-\mathrm{Z}(p)}{\hat{\mathrm{Z}}(p)}\right]^{2} d p\right]^{\frac{1}{2}}
\end{aligned}
$$

These relative indices are between 0 and 1.They are 0 in minimum difference and 1 in maximum distance.

We report the new GOF criterion based on distance between empirical and theoretical inequality curves for W, LN, and LL models for EU-SILC income data in Table 2. The lowest value of GOF indices are reported in bold type.

The results of Table 2 show that similar results are obtained with the new GOF measures. Since the values of GOF measures are smaller for LL model compared to other distributions, the results show that the LL model performs better fit than LN and W models in terms of the new GOF criteria. Since the proposed measure is a general measure, it is natural that the competitors also be general. The competitor tests are chosen from the class of tests discussed in D'Agostino and Stephens (1986). The test statistics of competitor tests are as follows: The Kolmogorov-Smirnov (KS), Cramer-von Mises (CH), Kuiper (V), and AndersonDarling (AD) test statistics are, respectively,

$$
\begin{aligned}
& \mathrm{KS}=\max _{1 \leq i \leq n}\left\{\frac{i}{n}-Z_{i}, Z_{i}-\frac{i-1}{n}\right\}, \\
& \mathrm{CH}=\frac{1}{12 n}+\sum_{i=1}^{n}\left(\frac{2 i-1}{2 n}-Z_{i}\right),
\end{aligned}
$$




$$
\begin{aligned}
\mathrm{V} & =\max _{1 \leq i \leq n}\left\{\frac{i}{n}-Z_{i}\right\}+\max _{1 \leq i \leq n}\left\{Z_{i}-\frac{i-1}{n}\right\}, \\
\mathrm{AD} & =-n-\frac{1}{n} \sum_{i=1}^{n}(2 i-1)\left\{\ln \left(Z_{i}\right)+\ln \left(1-Z_{n-i+1}\right)\right\},
\end{aligned}
$$

where $Z_{i}=\mathrm{F}_{0}\left(x_{(i)}, \hat{\theta}\right), i=1,2, \ldots, n$ and $\mathrm{F}_{0}$ is the cumulative distribution function under the null distribution.

In Table 3, we also report the general GOF criteria (KS, CH, V, and AD) for W, LN, and LL models for EU-SILC income data. The lowest values of GOF criteria are reported in bold type.

The findings of Table 3 confirm the results of Tables 1 and 2 . Since the values of these GOF measures are smaller for LL model compared to the two other distributions, then the LL model performs better fit than LN and W models.

For illustrative purposes and use our method to discriminate between the distribution functions, we refer to Dey and Kundo (2010). They considered the problem of discriminating between the LN and LL distributions to analyze lifetime data. They fitted the two distribution functions with the MLEs of the different parameters for deferment distribution functions. They analyzed the failure times of the air conditioning system of an airplane (in hours): 23, 261, 87, 7, 120, 14, 62, 47, 225, 71, 246, 21, 42, 20, 5, 12, 120, 11, 3, 14, 71, 11, 14, 11, 16, 90, 1, 16, 52, 95.

They showed from the log-likelihoods, K-S distances, and also from the $\chi^{2}$ values that the LN distribution is the preferred one for data set. The left panel of Figure 3 depicts the empirical Zenga curve for real data set and the theoretical Zenga curve for the LN income distribution. In the right panel of Figure 3, the corresponding $\mathrm{Z}(p)$ curves are drawn for LL model. The behavior of the Zenga curves show that the theoretical models overestimate the empirical distribution. Therefore, it is clear that based on the distance between the empirical and the theoretical Zenga curves, the LN model is the better model.

Table 3. The general GOF criteria for fitted models to EU-SILC income data

\begin{tabular}{rrrrr} 
Model & KS & CH & V & AD \\
\hline W & 0.0725 & 30.9790 & 0.1389 & 202.9310 \\
LN & 0.0682 & 21.6230 & 0.1119 & 140.5570 \\
LL & $\mathbf{0 . 0 1 7 2}$ & $\mathbf{1 . 1 5 6 9}$ & $\mathbf{0 . 0 3 2 1}$ & $\mathbf{1 5 . 8 7 9 6}$ \\
\hline
\end{tabular}




\section{A NEW GOODNESS OF FIT MEASURE}
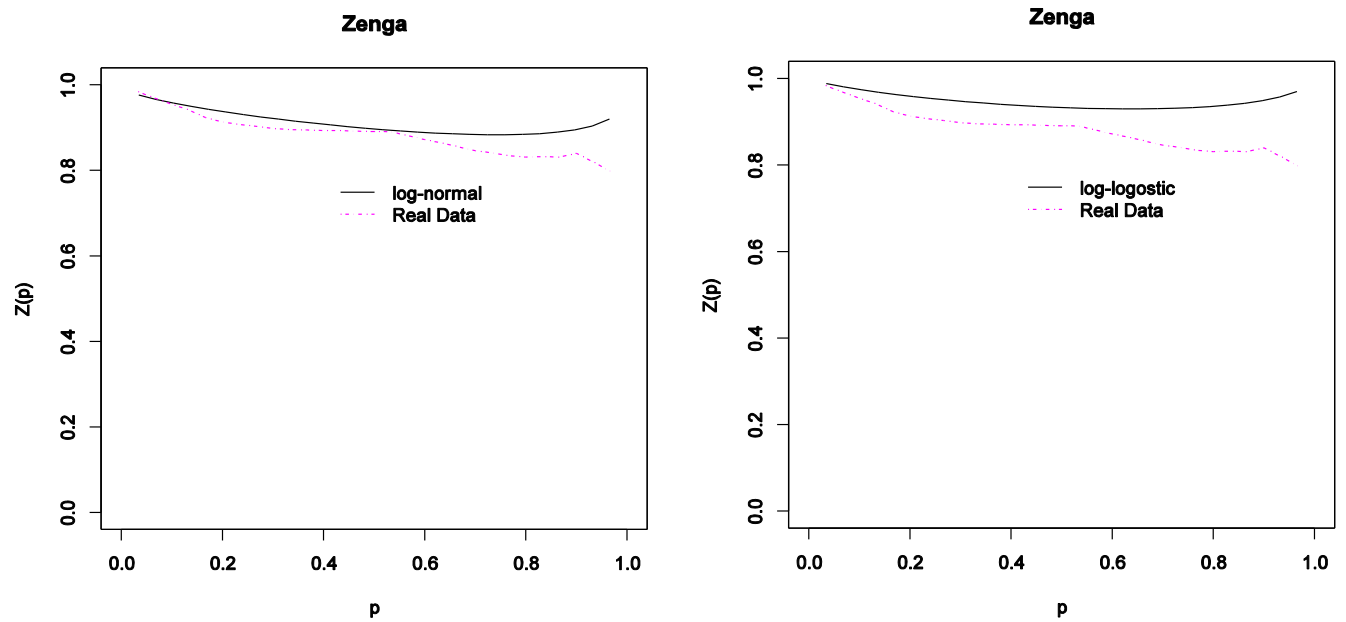

Figure 3. Zenga curves for empirical and fitted distributions for the failure times of the air conditioning

Table 4. The new GOF criteria for fitted models to the failure time's data

\begin{tabular}{rrrrr} 
Model & $\boldsymbol{B}_{1}$ & $\boldsymbol{B}_{\mathbf{2}}$ & $\boldsymbol{B}_{3}$ & $\boldsymbol{B}_{4}$ \\
\hline$L N$ & $\mathbf{0 . 0 3 8 1}$ & $\mathbf{0 . 0 3 5 9}$ & $\mathbf{0 . 0 4 9 6}$ & $\mathbf{0 . 0 4 5 5}$ \\
$L L$ & 0.0796 & 0.0722 & 0.0914 & 0.0811 \\
\hline
\end{tabular}

Here, we provide the new GOF measures based on Zenga curves for LN and LL distributions in Table 4. In this case based on the new GOF values, between the two distribution functions, clearly LN is the better choice.

The results show the consistency between these new GOF measures and the other popular GOF criteria. They approach the GOF in different ways. For this reason, the distance between inequality curves and the related GOF criterion seem to be valid alternative to the other common GOF measures.

\section{Simulation Results}

This section presents the results of a Monte Carlo experiment designed to measure the values of the new indices introduced in the previous section in finite samples. To do this, we have assumed as a model for the size distribution of incomes the generalized beta of the second kind (GB2) (McDonald \& Xu, 1995), which is very 


\section{MIRZAEI \& JAHANSHAHI}

flexible, with the ability to take a wide variety of shapes depending on particular values of its parameters. Its density is

$$
\mathrm{f}_{\mathrm{GB} 2}(x ; a, b, p, q)=\frac{a x^{a p-1}}{b^{a p} \mathrm{~B}(p, q)\left[1+\left(\frac{x}{b}\right)^{a}\right]^{p+q}},
$$

with $x>0, a, b>0$ and where $\mathrm{B}(p, q)$ is the Beta function given by

$$
\mathrm{B}(p, q)=\frac{\Gamma(p) \Gamma(q)}{\Gamma(p+q)}=\int_{0}^{\infty} \frac{t^{p-1}}{(1+t)^{p+q}} d t, \quad p, q>0 .
$$

This model nests many distributions as special or limiting cases including, among others, the beta of second kind (B2), Burr XII or Singh-Maddala (SM), Dagum (D), Lomax (Lom), Inverse Lomax (ILom), lognormal (LN), Weibull (W), Pareto, Fisk or log-logistic, and exponential (Exp). The details of many of these relationships are summarized in McDonald and Xu (1995) and Kleiber and Kotz (2003). Figure 4 provides a convenient visual summary of some limiting and special case of GB2 and their relationships.

In the following we will refer to Monte Carlo samples drawn from a GB2 distribution with scale parameter equal to $b=20933$ and shape parameters equal to $a=5.2, p=0.5$, and $q=0.77$ which closely mirrors the fitted distribution to EUSILC income data. It should be noted that the parameters considered are the maximum likelihood estimates of the GB2 distribution based on EU-SILC income data.

Here, we have performed an analysis on comparison the Bias and MSE of the new estimates in fitted distribution to real data. For better interpretation, the results have been shown in Table 5 .

It is evident that the four estimates are affected by positive bias, that is, they overestimate the values of parameters. From the results, we can verify that as the sample size increases, the mean estimates of the parameter tend to be closer to the true value, since bias and MSE decay toward zero. 


\section{A NEW GOODNESS OF FIT MEASURE}

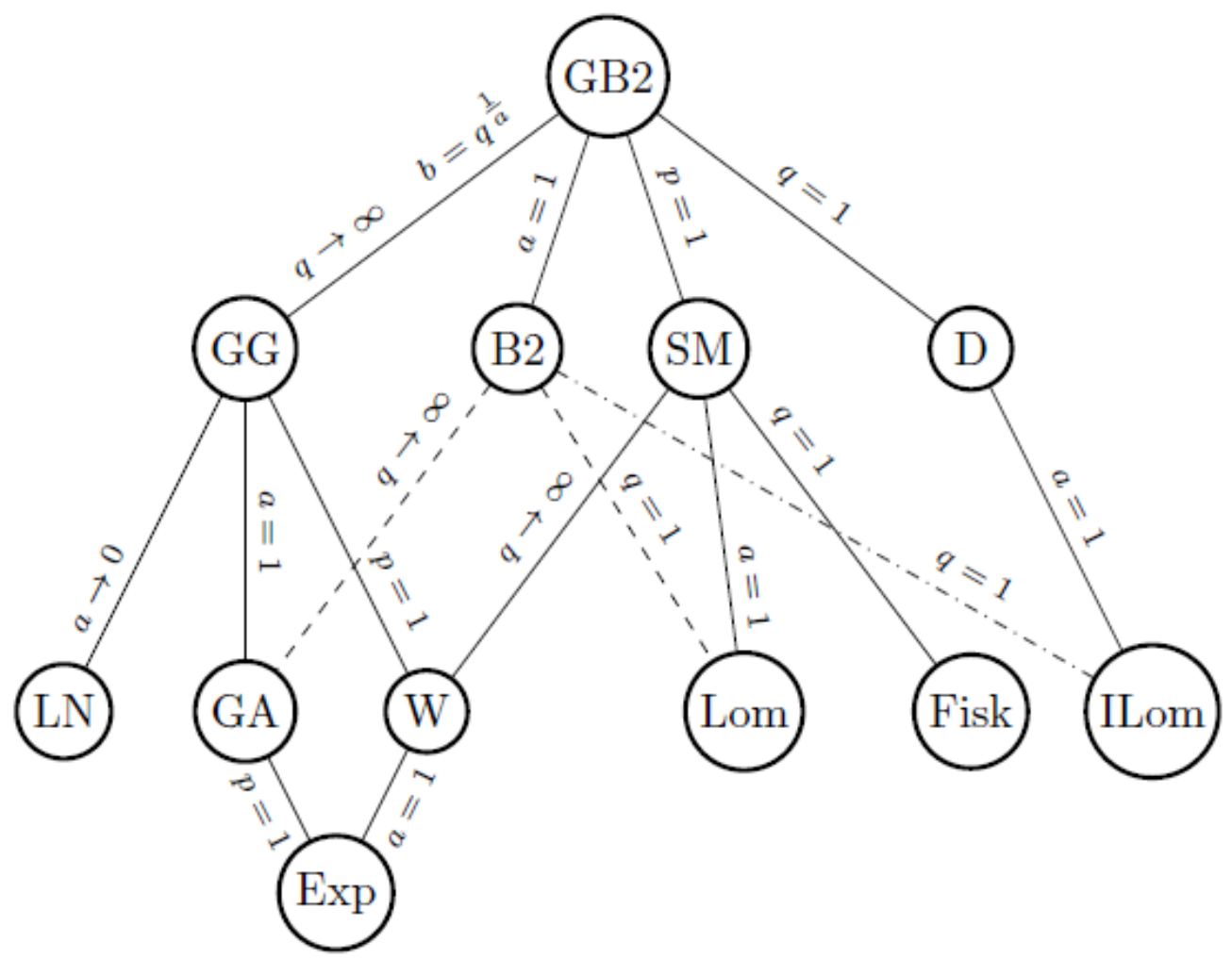

Figure 4. GB2 size distributions and their interrelations

Table 5. The comparison of Bias and MSE of the new indices based on Zenga curves

\begin{tabular}{|c|c|c|c|c|c|c|c|c|}
\hline \multirow[b]{2}{*}{$n$} & \multicolumn{2}{|c|}{$B_{1}$} & \multicolumn{2}{|c|}{$B_{2}$} & \multicolumn{2}{|c|}{$B_{3}$} & \multicolumn{2}{|c|}{$B_{4}$} \\
\hline & Bias & MSE & Bias & MSE & Bias & MSE & Bias & MSE \\
\hline 10 & 0.050671 & 0.010620 & 0.027881 & 0.007167 & 0.032821 & 0.055520 & 0.028882 & 0.054870 \\
\hline 20 & 0.025036 & 0.004340 & 0.018544 & 0.003534 & 0.015735 & 0.027544 & 0.032821 & 0.055285 \\
\hline 30 & 0.016714 & 0.002769 & 0.013247 & 0.002180 & 0.012060 & 0.018751 & 0.021571 & 0.035552 \\
\hline 50 & 0.010233 & 0.001640 & 0.008663 & 0.001314 & 0.009476 & 0.001234 & 0.003282 & 0.000552 \\
\hline 100 & 0.004873 & 0.000818 & 0.004097 & 0.000639 & 0.007543 & 0.000578 & 0.008328 & 0.000761 \\
\hline 500 & 0.001203 & 0.000120 & 0.001003 & 0.000178 & 0.009596 & 0.000119 & 0.001282 & 0.000142 \\
\hline
\end{tabular}



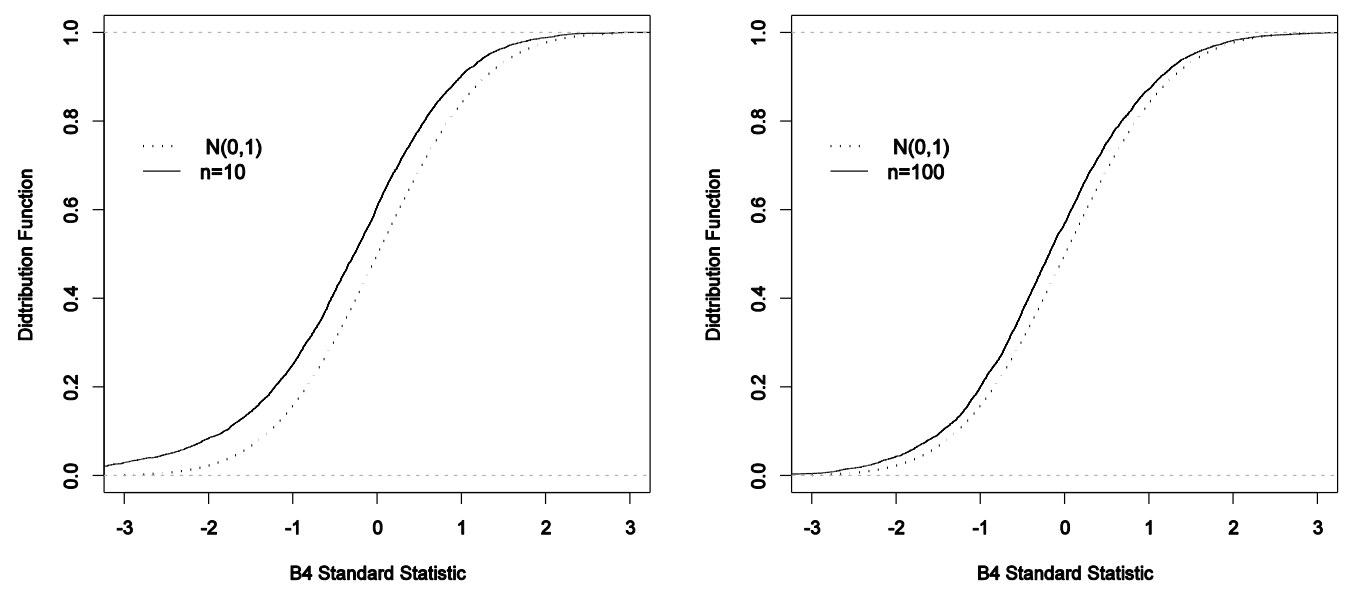

Figure 5. Distribution of $B_{4}$ standardized statistic as a function of sample size

Here, we study by simulation to what extent the $B_{4}$ estimator (as an example) proposed here give reliable inference. First, in order to see whether the asymptotic normality assumption yields a good approximation, simulations were undertaken with drawings from the fitted distribution to real data. In Figure 5, graphs are shown of the empirical distribution function of 10,000 realizations of the statistic

$$
\tau_{B_{4}}=\frac{\hat{B}_{4}-B_{4}}{\hat{\sigma}_{\hat{B}_{4}}}
$$

It can be noted that the estimation of the standard error was obtained using bootstrapping method. For sample sizes $n=10$ and 100 the graph of the standard normal cdf is also given as a benchmark in Figure 5. It can be seen that the new estimator is consistent and its asymptotic standard normal is good.

\section{Concluding Remarks}

We constructed a new measure of GOF by measuring distance between empirical and theoretical inequality curves. The new measure can be utilized with confidence because it has very simple meaning and interesting graphical representation. There is also the consistency between these new GOF measure and the other popular criteria. Furthermore, this new measure is bounded between 0 and 1 . A simulation 


\section{A NEW GOODNESS OF FIT MEASURE}

study based on fitted distribution to real income data shows that the plug-in estimator for the new measure is consistent and its asymptotic standard normal is

good. The results of this paper show that the new measure is a reasonable candidate for GOF criterion. We hope that the discussed measure may attract wider applications in econometrics and statistics.

\section{References}

Akaike, H. (1974). A new look at the statistical model identification. IEEE Transactions on Automatic Control, 19(6), 716-723. doi:

10.1109/TAC.1974.1100705

Arcagni, A., \& Porro, F. (2014). The graphical representation of inequality. Revista Colombiana de Estadistica, 37(2Spe), 419-436. doi:

10.15446/rce.v37n2spe.47947

Ascher, S. (1990). A survey of tests for exponentiality. Communication in Statistics - Theory and Methods, 19(5), 1811-1825. doi: 10.1080/03610929008830292

Bonferroni, C. E. (1930). Elementi di statistica generale. Libreria Seber, Firenze.

Cordeiro, G. M., Ortega, E. M., \& Ramires, T. G. (2015). A new generalized Weibull family of distributions: mathematical properties and applications. Journal of Statistical Distributions and Applications, 2(1), 1-25. doi: 10.1186/s40488-0150036-6

Cramér, H. (1928). On the composition of elementary errors: First paper: Mathematical deductions. Scandinavian Actuarial Journal, 1928(1), 13-74. doi: 10.1080/03461238.1928.10416862

D'Agostino, R. B., \& Stephens, M. A. (1986). Goodness-of-fit-techniques. New York: Marcel Dekker, Inc.

Dey, A. K., \& Kundu, D. (2010). Discriminating between the log-normal and log-logistic distributions. Communications in Statistics - Theory and Methods, 39(2), 280-292. doi: 10.1080/03610920902737100

Gini, C. (1921). Measurement of inequality of incomes. Economic Journal, 30(121), 124-126. doi: 10.2307/2223319

Jammalamadaka, S. R., \& Goria, M. N. (2004). A test of goodness-of-fit based on Gini's index of spacings. Statistics and Probability Letters, 68(2), 177187. doi: 10.1016/j.spl.2004.02.009 


\section{MIRZAEI \& JAHANSHAHI}

Kleiber, C., \& Kotz, S. (2003). Statistical size distributions in economics and actuarial sciences. New York: John Wiley. doi: 10.1002/0471457175

Lorenz, M. O. (1905). Methods of measuring the concentration of wealth. Publications of the American Statistical Association, 9(70), 209-219. doi: 10.1080/15225437.1905.10503443

Marsaglia, G. T., Sang, W. W., \& Wang, J. (2003). Evaluating Kolmogorov's distribution. Journal of Statistical Software, 8(18), 1-4. doi: 10.18637/jss.v008.i18

McDonald, J. B., \& Xu, Y. J. (1995). A generalization of the beta distribution with applications. Journal of Econometrics, 66(1-2), 133-152. doi: 10.1016/0304-4076(94)01612-4

Noughabi, H. A., Arghami, N. R., \& Borzadaran, G. R. M. (2014). A test of goodness of fit based on Gini index. Istatistik Journal of The Turkish Statistical Association, 7(1), 23-32. Retrieved from https://dergipark.org.tr/en/pub/ijtsa/issue/39090/458821

Polisicchio, M., \& Porro, F. (2009). A comparison between Lorenz L(p) curve and Zenga I(p) curve. Statistica Applicata, 21(3-4), 289-301. Retrieved from https://www.sa-ijas.org/rivista/en/Vol21num3-4.html

Watson, G. S. (1961). Goodness-of-fit tests on a circle. Biometrika, 48(1/2), 109-114. doi: $10.2307 / 2333135$

Zenga, M. (1984). Tendenza alla massima ed alla minima concentrazione per variabili casuali continue. Statistica, 44(4), 619-640.

Zenga, M. (2007). Inequality curve and inequality index based on the ratios between lower and upper arithmetic means. Statistica \& Applicazioni, 5(1), 3-27. 